Please quote as: Dickhaut, E.; Janson, A. \& Leimeister, J. M. (2021): The Hidden Value of Patterns - Using Design Patterns to Whitebox Technology Development in Legal Assessments. In: 16th International Conference on Wirtschaftsinformatik (WI 2021). University of Duisburg-Essen, Germany. 


\title{
The Hidden Value of Patterns - Using Design Patterns to Whitebox Technology Development in Legal Assessments
}

\author{
Ernestine Dickhaut $^{1}$, Andreas Janson ${ }^{2}$, and Jan Marco Leimeister ${ }^{1,2}$ \\ ${ }^{1}$ University of Kassel, Information Systems, Kassel, Germany \\ \{ernestine.dickhaut, leimeister\}@uni-kassel.de \\ ${ }^{2}$ University of St.Gallen, Information Systems, St.Gallen, Switzerland \\ \{andreas.janson, janmarco.leimeister\}@unisg.ch
}

\begin{abstract}
Higher legal standards with regards to data protection of individuals such as the European General Data Protection Regulation (GDPR) increase the pressure on developing lawful technologies. The development requires feedback from stakeholders such as legal experts that lack technical knowledge but are required to understand IT artifacts. As a solution, patterns can support interdisciplinary system development. We demonstrate how design patterns can support legal experts in arguing about technologies in court by introducing a law simulation study which is a well-known evaluation method in law. Our results show that patterns support legal experts in their argumentation about technologies in court. We provide theoretical contributions concerning cognitive fit theory about how patterns act as a bridge between the internal and external representation of problems and improve problem-solving performance related to the legal assessment of technology. In addition, we provide practical guidance for codifying and communicating design knowledge through patterns.
\end{abstract}

Keywords: Design Pattern, Law Simulation Study, Cognitive Fit Theory

\section{Introduction}

Socio-technical system development has become increasingly important, since not only the technical system is considered in isolation, but also the user and their environment $[1,2]$. When building information systems (IS), more and more disciplines like psychology, marketing, economics, law, and sociology are considered. Thus, not only software developers play a decisive role in the design and implementation but also lawyers and legal experts who deal with issues of legal aspects in information systems. Two factors are crucial in the development and assessment of lawful technologies, the development, and the legal assessment. On the one side, higher legal standards with regards to the data protection of individuals such as the European general data protection regulation (GDPR) are increasing the pressure on developers of IT artifacts [3]. In practice, it is often the case that the measures necessary to launch a system on the market are only considered at the end of the development process [4]. Usually, it happens with the least possible amount of attention so that the system just about meets the minimum requirements of the legal system. For example, due to the COVID-19

16th International Conference on Wirtschaftsinformatik,

March 2021, Essen, Germany 
situation, video conference systems such as ZOOM have become increasingly important. However, especially ZOOM was subject to major violations of the GDPR in the huge European market that ultimately led to extensive ad-hoc changes in the system functionalities as well as heavily revised privacy statements [5]. These technologies have to comply with legal requirements to avoid penalties. This could be prevented by paying sufficient attention to legal requirements in advance and in a systematic manner. Concerning the legal assessment related to the tradeoff between the quality of an IT artifact and its lawfulness, there is indeed a "legal limbo" [6]. Through questions of interpretation and the complex nature of legal aspects, there is also room for specific design decisions of IT artifacts that could be more or less compatible to legal regulations. To support the lawful system development a way must be found that support developers in understanding and implement legal requirements by capturing legal design knowledge and makes it accessible and applicable to developers [7].

On the other side, if there is a violation of the law, the IT artifact will be subject to court cases, and the lawfulness of the systems must be negotiated. Here, lawyers often lack technical knowledge and do not have the knowledge to investigate the background of the technology. In the legal assessment, lawyers use the information they get from their clients by using documents like contracts, reports, or documentation [8]. There is, to the best of our knowledge, no approach that supports lawyers in understanding complex socio-technical systems.

In the development of systems, (design) patterns are proven support for the development [9]. Patterns describe frequently occurring problems and outline the core of possible solutions [10]. In the form known so far, patterns usually support (only) developers in the implementation of technical problems by presenting possible solutions.

By providing patterns with legal and technical knowledge, i.e., patterns that make legal knowledge accessible for software engineers, the added value of patterns not only supports developers but also supports legal experts in understanding complex sociotechnical systems, e.g., to argue about technical facts in court cases but also a priori when assessing newly developed IT artifacts. The goal of our paper is to present an approach in which patterns also provide an added value for legal experts in their work dealing with IT systems and it is based on the following research question (RQ):

RQ: How can design patterns support legal experts in the assessment of complex socio-technical systems in court?

To answer our research question, we use a pattern catalog that considers legal and user experience requirements. With the help of the pattern catalog, a SPA [11] for the teaching context is developed. To investigate the use of the pattern catalog by giving legal experts an understanding of the development, we use the catalog as support for lawyers in court. For this purpose, we use a law simulation study, which is a wellknown evaluation method among law researchers for capturing the lawfulness of IT artifacts [12]. When considering a design science research (DSR) perspective on evaluation, we, therefore, maximize the summative and naturalistic evaluation perspective through the simulation study [12]. Thus, we contribute to theory by extending cognitive fit theory to improve a missed cognitive fit [13] between internal and external representation by using interdisciplinary patterns as a bridge to improve 
the understanding of the negotiated technology. In addition, we contribute to practice by deriving insights how far patterns support negotiation in a court case and whitebox the development of complex IT artifact, by making the procedure and the details of the development accessible to external parties.

\section{Related Work \& Theoretical Background}

\subsection{Application of Design Patterns}

In system development, design patterns document known and proven solutions to recurring problems [14]. In the literature, patterns contain templates to describe information in tabular form and represent established instruments to make complex knowledge accessible and applicable [15]. Thus, the use of patterns has become established in various disciplines. In Human-Computer Interaction (HCI), patterns have already been proven in many studies to teach design principles and design concepts [4, $16,17]$. Originally design patterns were used by Christopher Alexander in the area of architecture [9]. In system development, patterns were established first through the Gang of Four (GoF) [14]. In addition to the previously used application areas, patterns can be used to enable a broad understanding of periphery disciplines [18]. There are already approaches that map legal knowledge into patterns $[10,19,20]$. With increasing time and success, the scope of the application of design patterns has been expanded and new advantages were discovered (see table 1).

Table 1. Application Scenarios of Design Patterns

\begin{tabular}{cc}
\hline Application Context & Source \\
\hline Communication of complex concepts between designers & {$[21]$} \\
\hline Record and encourage the reuse of "best practices" & {$[21]$} \\
\hline Recurring design in building architecture & {$[9]$} \\
\hline Record and reuse existing design knowledge & {$[14]$} \\
\hline Teaching HCI design & {$[10,17,18]$} \\
\hline Best practice of programming languages such as "Smalltalk" & {$[22]$} \\
\hline Improving design skills & {$[23]$} \\
\hline Develop lawful technologies & {$[10,19,20]$} \\
\hline
\end{tabular}

Design patterns are an approach to codify design knowledge. Design knowledge is a special form of knowledge, namely, knowledge to design a system including methods and constructs [24]. As soon as design knowledge is codified for a group that differs in its expertise, further challenges arise. These challenges arise especially in interdisciplinary teams when considering socio-technical system development, which also encompasses engineering aspects that relate to legal aspects that we focus on in this paper. In this context, the codification of design knowledge is gaining importance, because members of an interdisciplinary team come from different disciplines that solve the same problem from different perspectives, with their own method and 
individual language, which harms knowledge sharing [25-27]. It should be guaranteed that the design knowledge is formulated in a clear, unambiguous, accessible language, and is free from inconsistencies and contradictions [28].

\subsection{Cognitive Fit Theory and Knowledge Transfer}

Cognitive fit theory was developed to understand how the fit between a task to be solved and the mental representation influences the skill to solve a problem [29]. A human characteristic is the abstract mental representation of situations and characteristics. Accordingly, the performance in solving a problem depends on the representation of the problem and the task. If there is a mismatch between both, the performance of problem solving a specific task will suffer [30]. Cognitive fit theory suggests that when both the problem representation and the problem to resolve correspond, a cognitive fit will occur. The cognitive fit produces a consistent mental representation for problem solving, and subsequently leads to faster and a more accurate performance in decisionmaking [31]. In recent decades, cognitive fit theory has been used to explain a wide range of problem-solving phenomena [13, 30, 32, 33].

The knowledge level is important and has an influence on the problem-solving performance. In interdisciplinary system development the level of knowledge and the transfer of knowledge gain in importance. In literature, differences regarding the level of knowledge due to different knowledge backgrounds exist such as levels of experience, and various disciplines are regarded as so-called knowledge boundaries, which must be resolved in the interaction of interdisciplinary teams [34]. Many studies have investigated how knowledge can be shared in interdisciplinary teams [34-38]. In the development of socio-technical systems, where many different disciplines come together, interdisciplinary cooperation is indispensable. Translations and interpretations between developers and other disciplines are still necessary [39]. By reusing and recombining knowledge, effectiveness, and efficiency can also be achieved, knowledge transfer of solutions to new use cases must be enabled [40]. Thus, we consider cognitive fit theory as a prime candidate for better understanding the value of patterns in complex socio-technical systems and scaffolding the subsequent theory development accordingly.

\section{Methodology: Law Simulation Study and Content Analysis}

The law simulation study is an established method among legal experts to evaluate technology in a practical manner in regard to their lawfulness [41]. A key characteristic is that it allows creating realistic conditions while damage is prevented. Therefore, it is desirable to provoke critical situations [12]. With the help of the simulation study, we are able to make statements about our goal to what extent the patterns make system development understandable for legal experts, besides contributing to a lawful system design. With the help of the simulation study, we are able to address legal requirements and assess the realization through the system before the system is launched to the market [42]. Therefore, we have the possibility to evaluate systems in an early state of 
implementation such as by evaluating prototypes. To our knowledge, it is currently the only known evaluation method that makes it possible to negotiate the lawfulness of technologies before they are launched to the market. The simulation study enables developers to receive legal feedback early (e.g. on prototypes). In addition, compared to legal opinion gathered a priori, the simulation study as an evaluation method reveals a richer picture related to the lawfulness of an IT artifact because it involves multiple rounds of negotiation between the stakeholders.

Simulation studies in general can be divided into two parts, starting with a user evaluation, and followed by a simulated court case. With the help of the design patterns, we have developed a smart personal assistant (SPA) which is used as a support in exam preparation as part of a course. The first part of the study evaluates the use with users, while the second part examines the lawfulness of the SPA in simulated court cases.

\subsection{Pattern Development and Pilot Study}

In the following, we want to provide a brief insight into the development of the pattern catalog as well as into the execution of a pilot study, in which developers evaluated the patterns regarding aspects such as the understanding, application, and usefulness.

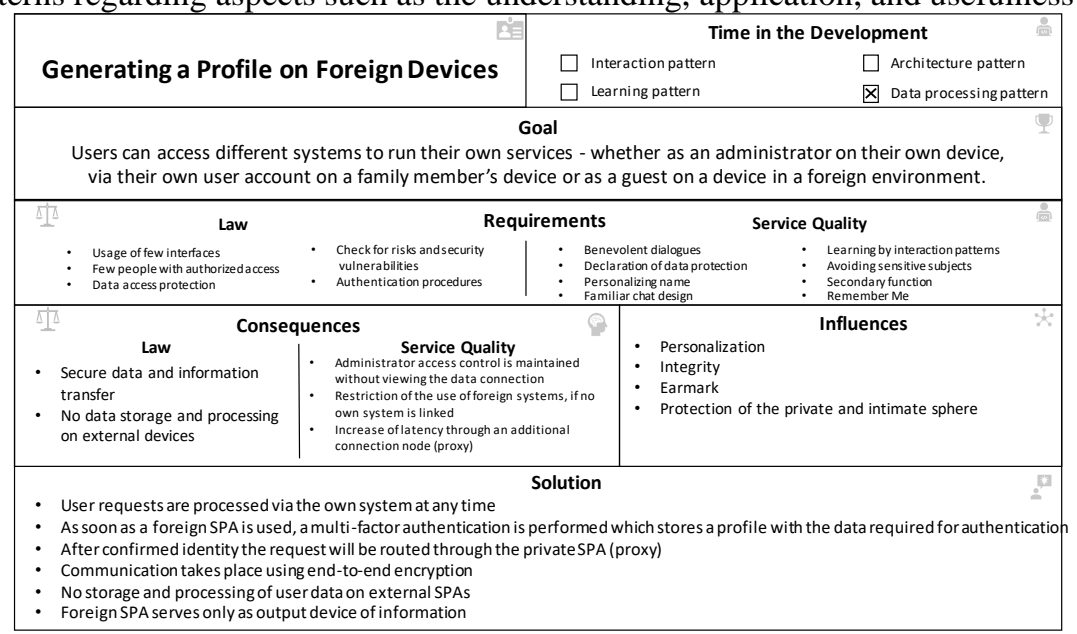

Figure 1. One exemplary design pattern

The development of the patterns was carried out in an iterative process consisting of several iterations to evaluate the patterns at an early stage and incorporate feedback. In a first step, a research team consisting of legal experts and computer scientists conduct literature reviews to investigate recurring issues that occur in the development of lawful SPAs. In addition to the theoretical insights, they conduct a workshop with developers $(\mathrm{N}=6)$. Based on the insights acquired through literature they prepare an overview of issues to match them with practical problems in the development. This procedure makes it possible to compare the results of the literature review with practice.

Based on the list of issues, the research team worked on proven solutions in the literature. In a second workshop with the practitioners, they extend the proposed 
solutions resulting in an overview of eleven issues and corresponding solutions at the end. The codification of the design knowledge into the patterns (see Figure 1) was carried out by an author of the paper. Based on a literature review on the codification of design knowledge and theories, such as cognitive load theory, the results were codified in patterns [43].

To make sure that the pattern developers support developers in developing lawful technologies, we have conducted a pilot study. In a $2 \times 2$ fully randomized field study we used manipulation to investigate how the support of patterns in the development of prototypes affects the assessment of legal experts. The results show that the group that was supported by pattern led to significantly better ratings of lawfulness [43].

For the user evaluation, we have used an IT artifact, which we developed in another study using interdisciplinary patterns [43]. The IT artifact is a voice-based conversational agent for exam preparation in university courses.

\section{2 $\quad$ First Part - User Evaluation}

The primary goal of the first part of the law simulation study is the use of the SPA by real users, similar to a usability evaluation [12]. This procedure enables the generation of legal violations by using the system in practice, as they might occur in reality. Therefore, violations of law are simulated for the later court case. Particular attention is paid to the fact that the violations have only been simulated and do not occur during the first part by using technology to avoid real damage. Nevertheless, the simulation of the violations is carried out as close to reality as possible to get a real situation.

In the user evaluation, we let students in a basic course for economics and business administration use the conversational assistant for half an hour a day for one week to revise the course material before the upcoming final assessment. Therefore, we prepared the teaching material to include it in the SPA. The teaching material is prepared as a flashcards quiz to make it as comprehensible and supportive as possible. The subjects use the SPA within the given time period and then give feedback on the use in questionnaires. This enables us to draw two conclusions. First, we can early evaluate the usability and user experience and improve it before launching on the market. On the other hand, a trial of the technology in case of an emergency is conducted, which helps us to improve the technology before market entry. During the user evaluation, legal experts observed the use and created four cases that could lead to court action. These cases are now used in the second part of the simulation study as a basis for the court cases.

\subsection{Second Part - Court Cases}

Based on the user evaluation, we have simulated four court cases. The simulation study was carried out before German courts according to German and European law. Overall, six legal experts participated in our law simulation study. Among them were two judges and four lawyers who conducted the four court cases. All participants have completed the second state examination in law and already have several years of professional experience as a lawyer or judge. One participant was female, the other five males. The 
oral hearings lasted 45 and 60 minutes. All participating lawyers received the patterns used in the development and a note that they were implemented in the development. The judges in the court cases, on the other hand, only received the evidence and material that was contributed to the court by the lawyers (as it would be in reality).

Two cases were heard before the civil court and two before the administrative court. To get a general impression of the support of our patterns, we conducted the civil cases in written form and the two administrative law processes in oral form. Each of the four trials involved a judge, a lawyer from the defendant's side, a lawyer from the plaintiff, the plaintiff, and the defendant. As plaintiffs, we recruited voluntary participants of the first part of the simulation study to present the process as realistically as possible. In all four cases, the defendant's side was represented by the university, which used the IT artifact in the lecture course.

In preparation for the oral hearings written preliminary proceedings took place. In a seven-page written pleading, the plaintiff's lawyer set out the facts of the case and the reasons for the action and called on the defendant to refrain from using the IT artifact in university teaching. The reason for one of the four actions was the collection of personal data beyond the purpose of processing, as well as information about the duration and purpose of data storage. In a five-page statement of defense, the defendant's lawyer commented on the action. In the statement of defense, the lawyer refers to the patterns that were used in the development process of the IT artifact.

The judge invites to an oral hearing to dispute the action. To be able to answer questions regarding the development of the IT artifact an expert who was involved in the development of the SPA. The expert leaves the courtroom before the start of the hearing and only joined to answer questions about the development. According to the administrative court rules, the oral proceedings began (after the case was called,) with the presentation of the essential content of the files. The judge first presented the facts of the case and discussed the reasons for the action. After the plaintiff's lawyer confirmed the facts of the case and set out the grounds of the action in more detail, the two lawyers and the judge examined the facts. Both parties now had the opportunity to present their side and the judge could get an impression of the situation. The negotiations end with the pronouncement of a judgment.

In addition to the four court cases and the written correspondence, we interviewed the judges and lawyers to gain insights into the support for our pattern catalog. The interview took place at once after the end of the simulation study with all participants. The interview allowed the participants to exchange views on the use of the patterns as well as to extract and discuss critical aspects necessary for the revision of the patterns. The advantage of conducting a group interview is that the participants can address aspects of the others. These insights allow us to draw a few conclusions about the added value of the patterns for the legal experts.

\subsection{Qualitative Content Analysis}

Through the simulation study, we received documents (see figure 2) from four court cases, which we examined in a structuring qualitative content analysis according to Mayring [44]. With the results of our analysis, we want to gain insights into how legal 
experts experienced working with interdisciplinary patterns to better understand how complex socio-technical systems can be designed. For this purpose, we have inductively formed categories based on our insights and the documents of the simulation study.

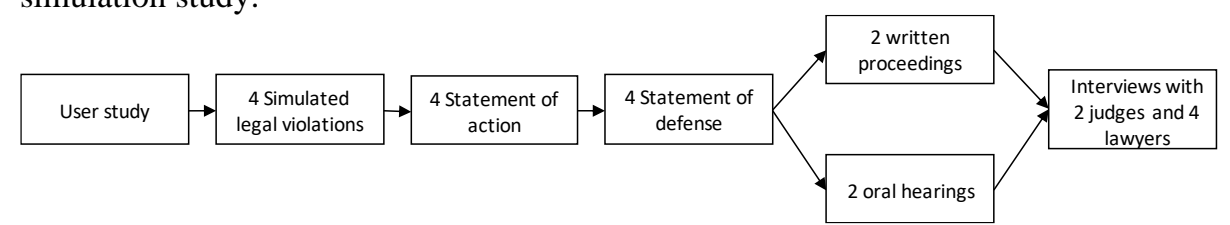

Figure 2. Procedure and Screened Documents

As a result, the two categories technical understanding and supporting argumentation emerged. Based on the categories, we have categorized our qualitative data. The data includes 1) two transcripts of the oral proceedings 2) the related correspondence between the lawyers and the judge before both oral proceedings 3 ) the documents of both written proceedings, and 4) the interviews of the lawyers and judges after the hearings. In the first step, paraphrasing, we cut out all the text components that are not content bearing. We have generalized the resulting statements and thus combined redundant statements into one common statement. In a second reduction, we summarized similar statements and combined them into general statements.

\section{$4 \quad$ Findings and Theoretical Propositions}

Regarding the intention of a qualitative content analysis, we screened our documents, and inductively formed two core categories in which the patterns were used, namely: understanding technical mechanisms, and supporting argumentation. Using the insights, we want to get detailed conclusions about how legal experts use the patterns in court. The first category technical understanding shows that legal experts support using patterns to get a better understanding of the technology to be negotiated (see table 2). In court, the lawyers and judges argue about an action of a technology to clarify the state of affairs. Each of the two lawyers pursues its own search for a solution to the problem. The plaintiff's lawyer argues against the use of the technology, while the defense lawyer argues why the technology did not lead to any violations of the law. The judge uses both arguments and tries to understand the technology to come to a judgment. For all parties, this means that the problem representation is formed from the action, the understanding of the technology, and the previous knowledge of the problem domain. To solve the problem the lawyer uses his mental representation about the problem domain together with the external representation about the technology. According to cognitive fit theory, a cognitive fit appears if each information matches.

Through further information and explanations in the pattern that goes beyond the technical solution, the contents of the pattern can be applied to the practical case (all following quotes are translated to English): 


\section{"[...] to meet the secondary burden of explanation, I have described the application and used the pattern." lawyer 1 (L1)}

The patterns are used to refer to the development in the argumentation and to be empowered to describe actual facts from the technology. The clear and uniform presentation of the patterns means that the necessary information can be found directly. Formulations that require no technical background make the patterns used stand out from technical documentation. These are difficult to understand and therefore offer little basis for developing a technical understanding:

"The technical information in the pattern is easy to understand, even for laymen with no technical background. " lawyer 4 (L4)

Table 2. Use of the Patterns in Court Cases

\begin{tabular}{c|c|c} 
Category & Reduction from the content analysis & Participant \\
\hline \multirow{4}{*}{$\begin{array}{c}\text { Technical } \\
\text { Understanding }\end{array}$} & Information goes beyond technical solution & L1, L3, L5 \\
\cline { 2 - 3 } & Applicable in court & L1, L3, L5 \\
\cline { 2 - 3 } & Reference to the development in the argumentation & L2 \\
\cline { 2 - 3 } & Supporting secondary burden of proof & L3, L5 \\
\cline { 2 - 3 } & Support the understanding of the technology & L1, L2 \\
\cline { 2 - 3 } & Clear, uniform presentation & L1, L4, J2 \\
\hline \multirow{4}{*}{$\begin{array}{c}\text { Supporting } \\
\text { argumentation }\end{array}$} & No need for technical background & L4, L3, J2 \\
\cline { 2 - 3 } & Linking law and technology & J1, L4 \\
\cline { 2 - 3 } & Support to find arguments & L1 L3 \\
\cline { 2 - 3 } & Offer helpful information & L1 \\
\cline { 2 - 3 } & Negotiation on technical basis & L3
\end{tabular}

Nevertheless, the possibility remains to use documentation as an addition to the patterns to get a more detailed insight into the development of the system. Through additional information, such as user stories and examples, the content of the patterns is not misunderstood and does not lead to false statements in court. The links to other additional patterns and additional information in the patterns are specially mentioned. With the help of links to other patterns and influencing factors, the contents can be applied to practice and be understood as a whole. Hence, we propose that further information in design patterns support the understanding of the technology.

Proposition 1 (P1): Design patterns act as a bridge between internal representation and external representation and contribute to a better problem representation, which can be used for the problem-solving task.

The second category supporting argumentation summarizes statements about the support in the formation of arguments and the justification based on the knowledge gained from the patterns in the court case. By linking law and technical requirements, the patterns show the conflict between user experience and law and that an attempt was made to meet the needs of both. In this way, a link to legal implementations in the technology can be drawn from the patterns in the case of technical points of attack. 
"The patterns are, in the end, aid for finding the argumentation." lawyer 3 (L3)

"They offer information to write a statement of defense." lawyer 2 (L2)

Especially in situations where background knowledge and understanding are missing, it is difficult to find convincing arguments. This is where patterns come in and provide the basis for the formation of arguments about the development that are nevertheless understandable. Because the other side's lawyer also understands the technical arguments, negotiations can be continued on a technical basis.

"The fact alone, that the pattern has been taken into account in the development shows the importance of protection of personal data in the development."

The use of the pattern already shows that the will was generally there to develop a lawful system. This could be used to the advantage of the defendant's technology, especially at the beginning of the trial. When it comes to negotiating fines, it is often a question of whether the person in charge has even thought about it:

"Here you can explain the first step, which means that the fines will be reduced. The more concretely one can then explain this, the better the argumentation." judge 1 (J1)

Proposition 2 (P2): Design patterns contribute to a comprehensive mental representation of the problem domain, thus expanding the space of possible solutions.

The interdisciplinary pattern catalog supports the formation of arguments during the trial but does not serve as independent evidence. The confirmation that the pattern has been implemented in the system completely without deviations is missing. With little time in the negotiation to react to arguments from the other side, the patterns must quickly provide exactly the information that is needed at that moment. Due to the clarity and the fact that all patterns correspond to the same structure, you can quickly get the used information and build arguments. The link between law and technology leads to an understanding of how the system functions technically, and provide an understanding of which legal requirements have been observed, and use it for argumentation:

"[...] you can see that the instructions were followed to implement legal requirements and argue with it." lawyer 1 (L1)

Proposition 3 (P3): Design patterns enable to through clear presentation of solutions to achieve a fit between problem representation and problem-solving task that, ultimately, leads to a better mental representation of a possible task solution.

Besides insights into the usefulness and comprehensibility of the patterns, we have also gained insights into the situations in which the lawyers used the patterns in the negotiation. At the oral hearing, arguments were presented on the basis of five patterns. In the second oral hearing, five patterns were used throughout the court proceedings for the argumentation. In both written procedures' patterns were also used to illustrate the development of the lawful IT artifact. Six patterns were used in the third process. While in the fourth process, six patterns were used to generate arguments. To show the use of 
the patterns, we use an extract from an oral hearing to show which arguments were used in the respective legal dispute (see table 3 ).

Table 3. Pattern as Support for Evidence

\begin{tabular}{|c|c|c|}
\hline Phase & Issue & Evidence \\
\hline Action & Data storage & Data protection policy \\
\hline \multirow{11}{*}{ Defense } & \multirow{11}{*}{$\begin{array}{c}\text { Data } \\
\text { minimization } \\
\text { principle Art. } 5 \\
\text { sect. } 1 \text { lit. c. } \\
\text { GDPR }\end{array}$} & Statement of expert \\
\hline & & Pattern "Data protection-friendly user profile" \\
\hline & & Pattern Differentiated purposes of use" \\
\hline & & Pattern "Non-linkability" \\
\hline & & Pattern "Prevention of personal data" \\
\hline & & Pattern "Deleting routines" \\
\hline & & Pattern "Transparent data processing procedures" \\
\hline & & Pattern "Setting options by the user" \\
\hline & & Pattern "No complete user profile" \\
\hline & & Pattern "Learning through relevance assessments" \\
\hline & & Pattern "Learning through interaction patterns" \\
\hline \multirow{9}{*}{ In Court } & \multirow{2}{*}{ Data storage } & Pattern "Deleting routines" \\
\hline & & Statement of the expert \\
\hline & \multirow{3}{*}{$\begin{array}{l}\text { Cancellation } \\
\text { right }\end{array}$} & Pattern "Data protection friendly user profile" \\
\hline & & Statement of the expert \\
\hline & & Data protection policy \\
\hline & \multirow{4}{*}{$\begin{array}{l}\text { Storage } \\
\text { purpose }\end{array}$} & Pattern "Differentiated purposes of use" \\
\hline & & Pattern "Prevention of personal data" \\
\hline & & Pattern "Setting options by the user" \\
\hline & & Pattern "No complete user profile" \\
\hline
\end{tabular}

The court case consists of a written preliminary hearing, in which the claim and defense are exchanged, and the oral hearing occurs. The key reason for the action is data minimization which supposedly has not been complied with. In his statement of defense, the lawyer refers to ten patterns:

"The design pattern 'privacy-friendly user profile' proposes that only data that are necessary to [...] should be stored. It is also recommended to give the user the possibility to decide which data may be processed." lawyer 1 (L1)

The reference to the design pattern enables the lawyer to shift the discussion from a legal argumentation level to a technical level. While the hearing has previously focused on the data minimization principle art. 5 sect. 1 lit. c. GDPR, the lawyer was able to show with the help of the design pattern that data minimization was generally taken into account and he has the possibility to show how this was implemented in the technology.

Proposition 4 (P4): Design patterns contribute to the extension of existing domain knowledge and for the acquisition of new knowledge. 
To support his argument, he mentions further patterns in his argumentation. Due to the change from the negotiation of purely legal aspects to the technical implementation of the legal requirements, the judge sees no need to discuss general questions about the extent to which data minimization was implemented. All further questions afterward relate to the question of whether the patterns mentioned were actually implemented in the technology in this way. The lawyer can confirm this with the following statement:

"These proposed solutions from the pattern "Data Protection Friendly User Profile" were fully considered and implemented when programming [...]." lawyer 1 (L1)

In the end, the judge invites the expert who should confirm that the mentioned patterns have really been implemented. All further questions refer to the actual implementation of the pattern in the technology.

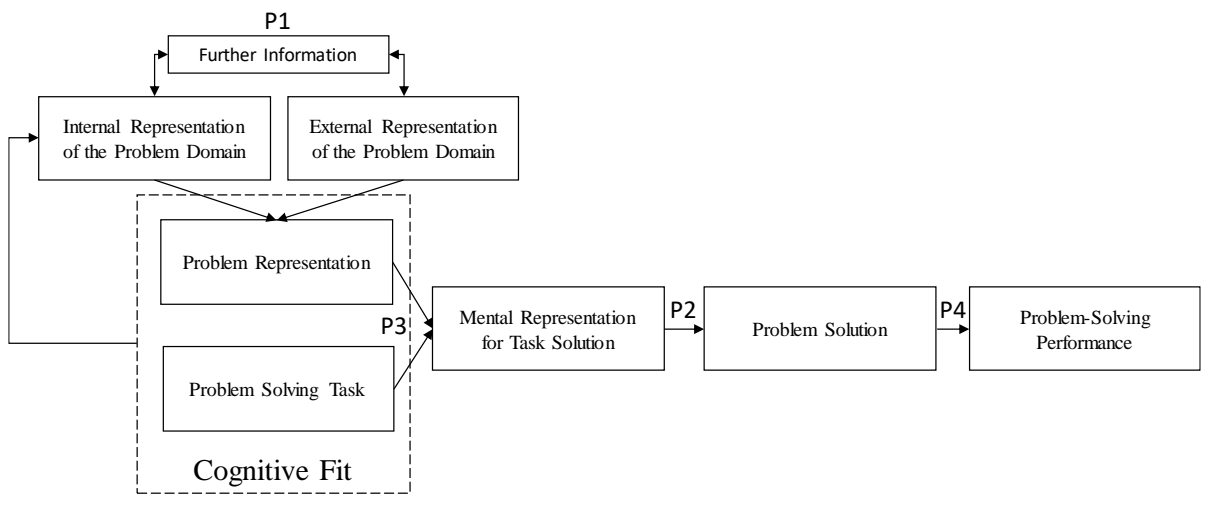

Figure 3. Design Pattern and Cognitive Fit Theory (adapted from [13])

Based on the findings we consider a cognitive fit between the understanding of a technology to be negotiated (mental representation) by legal experts and the clarification of the facts (problem-solving task) to be a decisive factor for a negotiation on the lawfulness of a technology in court (P3). The mental representation consists of the internal representation of the problem domain, i.e., the existing knowledge about the technology to be negotiated, and the external representation of the problem, the technology itself. As in practice legal experts often lack the necessary domain knowledge on complex socio-technical systems hence the internal presentation of technical domain knowledge that can be accessed is limited. In our case, the external presentation of the technology consists of technical documentation and programming code which is difficult to understand for legal experts and difficult to use for negotiation. Therefore, we see the extension of a bridge between the internal and external representation in the extended cognitive fit model of [33] as a crucial factor in building the cognitive fit and thus improving problem-solving performance. We see an opportunity (see figure 3 ) in which the cognitive fit can be produced with the help of further information (in our case the use of interdisciplinary patterns).

A crucial point that must be taken into account is that the person is aware of a mismatch and lack of cognitive fit and realizes this amongst other things in the fact that 
he cannot solve the task this way. They then look for information that help them to solve the task (P1). The information acts between the internal and external representation $(\mathrm{P} 2)$ of the problem and bridges the mismatch of understanding, leading to a mental representation that leads to a problem solution. At any time, when new information is added to the internal representation, the mental representation is compared with the task to be solved and it is decided whether a) further information is needed or b) the problem can be solved by the circumstances of the mental representation. Our simulation study shows the benefit in legal patterns that act as a bridge between the internal and external representation by codifying and accessing design knowledge from different domains in a layman's language to improve the negotiation in court (P4).

\section{Discussion}

\subsection{Theoretical and Practical Implications}

Our findings show that the use of interdisciplinary design patterns in the context of court cases increase the understanding of technical mechanisms. A connection between legal requirements and influences on the technical solution is mapped, which offers links between law and development.

Hong et al. [30] argues that the same type of representing the solving task and the mental representation is crucial for the cognitive fit. According to [30] we assume that a bridge between the internal and external representation can support the cognitive fit because both representations should be on the same professional level of the domain knowledge. By formulating the interdisciplinary patterns in a layman's language, they help the legal experts to understand, (build a mental representation of the technology [29]) and support the possibility to negotiate the technology. For example, technical documentation of the code is usually poorly understood by lawyers and cannot be used to understand the problem domain, which would lead to no cognitive fit [31].

From a design science theoretical perspective, patterns provide a means to the end for accumulating design knowledge of IT artifacts in a way that is comprehensible enough to not only build IT artifacts but also communicate IT artifact design effectively across disciplines [14]. Accordingly, patterns are a carrier of design knowledge, which serves as a mediator between developers and legal experts by acting as a bridge between the external representation (technology itself) and the internal representation (legal experts' knowledge of the technological domain). Thus, on the one hand, design patterns provide guidance on the code and technical implementation [9]. On the other hand, lawyers and judges are trained in technical understanding in order to negotiate the state of affairs in court.

Our findings indicate the added value of patterns in two cases, the development and the legal assessment. In both cases the patterns act as support. In the development the patterns support the legal understanding of the developer and provide proven solutions for recurring legal problems. 


\subsection{Limitation and Future Research}

Our study is limited by a few factors that provide directions for future research. First, by having evaluated the use of the patterns in a natural scenario, the results can only be generalized to a limited extent. The methodology of the simulation study has the limitation that a technology is evaluated extensively, but with a small sample size. At the evaluation in court, seven legal professionals were asked and observed, which does not offer a strong evidence base. Nevertheless, we have provided a first insight into possible scenarios of patterns in the work of legal experts. We see a need for further research, which, among other things, will examine the usefulness of a larger sample.

Second, the fact that our infringements are based on the user evaluation of the simulation study means that they are invented. Thus, it cannot be guaranteed that exactly these cases would happen in reality. In preparing the legal infringements, we have used years of practical experience of lawyers who have extracted simulated legal infringements to the best of their knowledge. Nevertheless, there is a bias that must be taken into account in any case.

Third, the legal validity of the patterns as evidence could lead to the fact that now everyone designs interdisciplinary patterns and refers to them in case of an action. The problem of developing lawful technologies is the interpretation of the law. Law is technology-neutral which leaves room for interpretation. The lawfulness of a technology has to be reconsidered in each individual case. Therefore, patterns act as a support to provide information and solutions but are no guarantee to develop lawful technologies. To close the gap between the development and the legal assessment we include a field in which the implementation can be confirmed. To prevent a large number of legal patterns from being created that claim to help design legal technologies, we see the need for certification of the patterns. Further work could, therefore, deal with a certification of legal patterns. In particular, the content should be checked for its correctness to support developing lawful technologies. Additionally, the interpretability of the patterns should not leave too much room for negotiation so that the patterns cannot be interpreted the wrong way. Further work in this area could, for example, be oriented towards certification types for medical technology and use their experience. This is the only way to guarantee that the expansion of patterns, as we know it from system development, can be extended to other disciplines, such as law, and bring highquality added value.

Fourth, the method of simulation study we use is, in its current form, linked to the European legal system. In comparison to other legal systems, European law and especially the GDPR represent strict legal requirements. In particular, the protection of personal data is in focus and is strictly protected. Nevertheless, we see the necessity to use the interdisciplinary pattern catalog in other legal systems as well and to focus on its utility.

\section{Conclusion}

We present an approach in which interdisciplinary patterns provide an added value for legal experts in understanding complex socio-technical systems. The law simulation 
study enables us to gain practical insights into the work with the patterns. We use the interdisciplinary pattern catalog to support lawyers in their argumentation and evidence during court cases. So, we have the opportunity to study the process of using the pattern in a unique scenario. We investigate the use of the pattern catalog in a natural setting that would not be possible in a laboratory study. In addition, to gain insights into the support of the pattern to make the development of the IT artifact transparent for legal experts, the simulation study enables a statement about the lawfulness of the developed technology.

The socio-technical system development would benefit from the approach of combining a two-sided added value from patterns, especially with regard to higher legal standards for data protection of individuals such as the GDPR. So far, the process of how systems were developed and what thoughts developers had in mind for lawyers has remained a black box, which makes it difficult to argue in court. This issue is especially prevalent when considering new AI-based technologies due to their complexity and blackbox character [45]. Technical documentation and further technical explanations would mostly be difficult to understand for laymen and, therefore, not sufficient for the formation of argumentations. In the development of socio-technical systems, various stakeholders are becoming increasingly relevant, which is why it is important to codify the meaning of design knowledge. Since, in practice, there is often a lack of exchange between the individual disciplines in the development of sociotechnical systems, we see the use of interdisciplinary patterns to create knowledge bridges as useful.

\section{Acknowledgements}

Our thanks go to all participating lawyers and judges without whose support the study would not have been possible. This paper presents research that was conducted in context of the project AnEkA (project number: 348084924), funded by the German Research Foundation (DFG).

\section{References}

1. Hoffmann, A., Söllner, M., Fehr, A., Hoffmann, H. \& Leimeister, J. M.: Towards an Approach for Developing socio-technical Ubiquitous Computing Applications (2011)

2. Sarker, S., Chatterjee, S., Xiao, X., Elbanna, A.: The Sociotechnical Axis of Cohesion for the IS Discipline: Its Historical Legacy and its Continued Relevance. MISQ 43 (2019)

3. Kühling, J., Martini, M.: Die Datenschutz-Grundverordnung: Revolution oder Evolution im europäischen und deutschen Datenschutzrecht (2016)

4. Compagna, L., Khoury, P.E., Massacci, F., Thomas, R., Zannone, N.: How to capture, model, and verify the knowledge of legal, security, and privacy experts. International conference on Artificial intelligence and law, 149-153 (2007)

5. Security Week: www.securityweek.com/zooms-security-and-privacy-woes-violated-gdprexpert-says (accessed on June, 25st 2020) 
6. Scott, J.: In legal limbo: Post-legislative guidance as a challenge for European administrative law. common market law review 48 (2011)

7. Morse, W.C., Nielsen-Pincus, M., Force, J.E., Wulfhorst, J. D.: Bridges and Barriers to Developing and Conducting Interdisciplinary Graduate-Student Team Research. Ecology and Society 12(2): 8 (2007)

8. Morcón, C.R., García, J.P., Pizarro, J.A.S.: Knowledge management in a law firm. Journal of knowledge management (2000)

9. Alexander, C.: A pattern language: towns, buildings, construction. Oxford University Press (1977)

10. Compagna, L., El Khoury, P., Krausová, A., Massacci, F., Zannone, N.: How to integrate legal requirements into a requirements engineering methodology for the development of security and privacy patterns. Artificial Intelligence and Law 17, 1-30 (2009)

11. Knote, R., Janson, A., Söllner, M., Leimeister, J.M.: Value Co-Creation in Smart Services: A Functional Affordances Perspective on Smart Personal Assistants. JAIS (2020)

12. Pordesch, V., Roßnagel, A., Schneider, M.: Simulation Study Mobile and secure communication in Healthcare. DuD, 76-80 (1999)

13. Shaft, T.M., Vessey, I.: The Role of Cognitive Fit in the Relationship between Software Comprehension and Modification. Management Information Systems Quarterly (2006)

14. Gamma, E., Helm, Richard, Johnson, Ralph, Vlissides, J.: Design Patterns: Elements of Reusable Object Oriented Software. AddisonWesley Professional (1994)

15. Alexander, C.: The timeless way of building. Oxford University Press, New York (1979)

16. Borchers, J.: Teaching HCI design patterns: Experience from two university courses. CHI (2002)

17. Koukouletsos, K., Khazaei, B., Dearden, A., Ozcan, M.: Teaching Usability Principles with Patterns and Guidelines, 159-174 (2009)

18. Wania, C.: Exploring Design Patterns as Evaluation Tools in Human Computer Interaction Education. MWAIS (2019)

19. Hoffmann, A., Schulz, T., Zirfas, J., Hoffmann, H., Roßnagel, A., Leimeister, J.M.: Legal Compatibility as a Characteristic of Sociotechnical Systems. BISE 57, 103-113 (2015)

20. Yskout, K., Scandariato, R., Joosen, W.: Do Security Patterns Really Help Designers? International Conference on Software Engineering, 292-302 (2015)

21. Beck, K., Crocker, R., Meszaros, G., Coplien, J.O., Dominick, L., Paulisch, F., Vlissides, J.: Industrial experience with design patterns. Proceedings of IEEE 18th International Conference on Software Engineering, 103-114 (1996)

22. Roberts, D., Brant, J., Johnson, R.: A refactoring tool for smalltalk. Theory Pract. Obj. Syst. 3, 253-263 (1997)

23. Prechelt, L., Unger-Lamprecht, B., Philippsen, M., Tichy, W.F.: Two controlled experiments assessing the usefulness of design pattern documentation in program maintenance. IEEE Transactions on Software Engineering 28, 595-606 (2002)

24. Gregor, S., Hevner, A.R.: Positioning and presenting design science research for maximum impact. MIS Quarterly, 337-355 (2013)

25. Chandra Kruse, L., Seidel, S., Purao, S.: Making Use of Design Principles. DESRIST, $37-$ $51(2015)$

26. Chandra Kruse, L., Seidel, S.: Tensions in design principle formulation DESRIST, 180188 (2017) 
27. Phuwanartnurak, A.J.: Interdisciplinary Collaboration through Wikis in Software Development. IEEE, Piscataway NJ (2009)

28. Lukyanenko, R., Jeffrey, P.: Design Theory Indeterminacy: What is it, how can it be reduced, and why did the polar bear drown? JAIS, 1-59 (2020)

29. Vessey, I., Galletta, D.: Cognitive Fit: An Empirical Study of Information Acquisition. Information Systems Research 2, 63-84 (1991)

30. Hong, W., Tam, J.Y., Tam, K.Y.: The Effects of Information Format and Shopping Task on Consumers' Online Shopping Behavior: A Cognitive Fit Perspective. JMIS, 149-184 (2004)

31. Agarwal, R., Sinha, A.P., Tanniru, M.: Cognitive Fit in Requirements Modeling: A Study of Object and Process Methodologies. Journal of Management Information Systems 13, 137-162 (1996)

32. Claes, J., Vanderfeesten, I., Gailly, F., Grefen, P., Poels, G.: The Structured Process Modeling Theory (SPMT) a cognitive view on why and how modelers benefit from structuring the process of process modeling. Inf Syst Front 17, 1401-1425 (2015)

33. Khatri, V., Vessey, I., Ramesh, V., Clay, P., Park, S.-J.: Understanding Conceptual Schemas: Exploring the Role of Application and IS Domain Knowledge. Information Systems Research 17, 81-99 (2006)

34. Kotlarsky, J., van den Hooff, B., Huysman, M.: Bridging Knowledge Boundaries in CrossFunctional Groups: The Role of a Transactive Memory System. ICIS (2009)

35. Bittner, E.A.C., Leimeister, J.M.: Creating Shared Understanding in Heterogeneous Work Groups: Why It Matters and How to Achieve It 31, 111-144 (2014)

36. Carlile, P.R.: Transferring, Translating, and Transforming: An Integrative Framework for Managing Knowledge Across Boundaries. Organization Science 15, 555-568 (2004)

37. Linden, T., Cybulski, J.: Refining the Process of Sharing Problem-Solving Experience across Domain: A Hermeneutic Study. AMCIS (2006)

38. Winkler, M., Huber, T., Dibbern, J.: The Software Prototype as Digital Boundary Object: A Revelatory Longitudinal Innovation Case (2014)

39. Pawlowski, Robey: Bridging User Organizations: Knowledge Brokering and the Work of Information Technology Professionals. MIS Quarterly 28, 645 (2004)

40. vom Brocke, J., Winter, R., Hevner, A., Maedche, A.: Accumulation and evolution of design knowledge in design science research-A journey through time and space. JAIS (2019)

41. Roßnagel, A., Schuldt, M.: The Simulation Study as a Method of Evaluating Socially Acceptable Technology Design, 108-116 (2013)

42. Otto, P.N., Anton, A.I.: Addressing Legal Requirements in Requirements Engineering. 15th IEEE International Requirements Engineering Conference, 5-14 (2007)

43. Dickhaut, E., Janson, A., Leimeister, J.M.: Codifying Interdisciplinary Design Knowledge through Patterns - The Case of Smart Personal Assistants. DESRIST (2020)

44. Mayring, P.: Qualitative content analysis: theoretical foundation, basic procedures and software solution (2014)

45. Rahwan, I., Cebrian, M., Obradovich, N., Bongard, J., Bonnefon, J.-F., Breazeal, C., Crandall, J.W., Christakis, N.A., Couzin, I.D., Jackson, M.O., et al.: Machine behaviour. Nature 568, 477-486 (2019) 